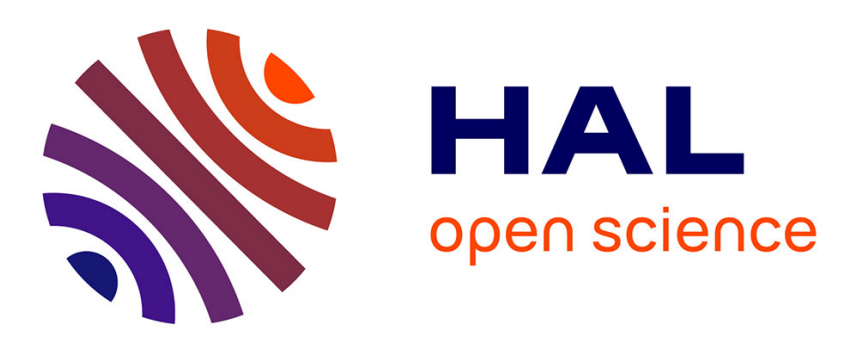

\title{
A unified approach based on temporal homogenisation and cycle jump for thermo-mechanical combined cycle
} fatigue

\author{
Mainak Bhattacharyya, David Dureisseix, Beatrice Faverjon
}

\section{To cite this version:}

Mainak Bhattacharyya, David Dureisseix, Beatrice Faverjon. A unified approach based on temporal homogenisation and cycle jump for thermo-mechanical combined cycle fatigue. International Journal of Fatigue, 2020, 131, pp.105320. 10.1016/j.ijfatigue.2019.105320 hal-02323165

\section{HAL Id: hal-02323165 \\ https://hal.science/hal-02323165}

Submitted on 20 Oct 2021

HAL is a multi-disciplinary open access archive for the deposit and dissemination of scientific research documents, whether they are published or not. The documents may come from teaching and research institutions in France or abroad, or from public or private research centers.
L'archive ouverte pluridisciplinaire HAL, est destinée au dépôt et à la diffusion de documents scientifiques de niveau recherche, publiés ou non, émanant des établissements d'enseignement et de recherche français ou étrangers, des laboratoires publics ou privés. 


\title{
A unified approach based on temporal homogenisation and cycle jump for thermo-mechanical combined cycle fatigue
}

\author{
Mainak Bhattacharyya $^{1, \dagger}$, David Dureisseix ${ }^{2}$ and Béatrice Faverjon ${ }^{2}$
}

${ }^{1}$ LMT Cachan, ENS Paris-Saclay, CNRS UMR8535, Université Paris-Saclay, F-94235, France

${ }^{2}$ Univ Lyon, INSA-Lyon, CNRS UMR5259, LaMCoS, F-69621, France

$\dagger$ Corresponding author

\begin{abstract}
Engineering components in the aviation, automobile and locomotive sectors are often subjected to thermo-mechanical fatigue of the CCF (combined cycle fatigue) type. The fatigue behaviour for such cases can be quantified using classical continuum damage framework. The problem in such case is the immense numerical cost for a cycle-by-cycle simulation, hence a unified numerical strategy is proposed that can avoid such tedious computations for structures under CCF. Such a framework is based on temporal homogenisation and cycle jump technique that avoids cycle-by-cycle simulation for both the fast and slow cycles, providing an appreciable CPU gain with acceptable accuracy.

This is a preprint of an article published in its final form as: Mainak Bhattacharyya, David Dureisseix, Béatrice Faverjon, A unified approach based on temporal homogenisation and cycle jump for thermo-mechanical combined cycle fatigue, International Journal of Fatigue, 2019

DOI: 10.1016/j.ijfatigue.2019.105320, Elsevier, licensed under CC-BY-NC-ND 4.0 International.
\end{abstract}

Keywords. combined cycle fatigue, cycle jump, homogenisation, multi-scale, thermo-mechanical, continuum damage mechanics

\section{Introduction}

Simulation of structures subjected to cyclic loading has been a bottleneck over the years, when a large number of fatigue cycles are involved (see [33]). For structures containing large number of degrees of freedom, with high amount of non-linearities, subjected to multi-physics loads (e.g. thermo-mechanical), cycle-by-cycle simulation becomes unaffordable. Although, there exists certain cumulative and empirical methods (see 15, 13, 35 for details) used for fatigue simulation in the industries, these do not consider the loading history and tend to deviate from accuracy for complex multi-physics problems. The interest of this article is in the initiation phase of the total fatigue life in the light of continuum damage mechanics (CDM).

Usage of continuum damage mechanics (CDM) initially introduced in 20 has been popular in fatigue simulation, as it defines a dedicated internal variable in a consistent thermodynamic framework to quantify the loss of load carrying capacity of a structure (see e.g. 10, 25, 21, 22, 24). The initiation phase of the total life can be estimated when this internal variable reaches its critical value. For kinetic damage models (damage as a function of time), it is difficult to reach a very high damage value especially when coupled with elasto-(visco)plasticity, and in case 
of thermo-mechanical problems with thermo-elasto-(visco)plasticity, due to loss of ellipticity. A way out can be the usage of non-local models (see [4, 1, 32, 19]) that prevents localisations of damage variables, thereby avoiding mesh-dependency, and high value of damage may be reached. Nevertheless, the focus herein will be more on the computational aspect and not on modelling, hence the concerned damage variable is the classical local one. Although it is possible to compute the initiation phase using CDM and then use crack propagation concepts to calculate the propagation phase (as done in [5]), fracture mechanics is beyond the scope of the current article.

A useful technique for fatigue simulation, first developed in [18 is the homogenisation technique, involving multi-scale description of time. This method has been used for classical elastoviscoplasticity in [31, 30, viscoelasticity in [36. As far as damage is concerned, it has been used for elasticity-based damage cases in [14], for GTN (Gurson-Tvergaard-Needleman) based damage models in [29, 17] and for a viscoplastility-like damage model in 28. In this article the case considered is a thermo-mechanical one, and the damage is driven by coupled thermoelasto-(visco)plasticity with mixed hardening. It is also interesting to observe that the loading cases considered in [2, 31, 30, 14] are of combined cycle fatigue (CCF) type, i.e. the macroscopic load is also cyclic containing high frequency microscopic fluctuations. The usage of homogenisation in such cases avoids cycle by cycle simulations of the micro cycles and provides extreme numerical benefit. However, the CCF type loading considered in the aforementioned articles contains very few macroscopic cycles and hence do not address cases containing large number of macroscopic cycles. In the current article, the interest is on CCF type loads involving large number of macroscopic cycles.

Another classical technique to avoid cycle by cycle simulation is the cycle jump method, where large number of cycles can be skipped based on the information of the last computed cycle, with the damage variable being linearly extrapolated. This technique was first introduced in [8, 27, 26] and different variants of it has been used in [25, 9, 12, 34. Similar technique has also been used for non-incremental iterative solvers like the LATIN method, for viscoplasticity (see [1]) for viscoplasticity coupled with damage (see [7]), and for two-scale damage models (see [6]). In the light of temporal homogenisation, a unified approach has been used in 16 for non-local damage models described by equivalent strains, where cycle jump has been used to skip macro time steps (i.e. microscopic cycle).

In the present article, a unified scheme is proposed for thermo-mechanical CCF, where cycle jump approach is applied for macroscopic cycles and temporal homogenisation is used to simulate a macro-cycle of interest. This will avoid cycle by cycle simulation for both macro and micro cycles. An attempt is made here to combine the advantages of both the individual strategies. Homogenisation by itself, although can be effectively accurate compared to cycle jump, however for highly non-linear problems, with highly non-linear macroscopic load variation, finer macroscopic temporal mesh is necessary to obtain such an accuracy. For CCF type loading involving large number of macro-cycles, such a temporal discretisation is not affordable and can be very expensive. Cycle jump alone is much cheaper compared to homogenisation, however employing jumping strategies alone on the macro-cycles will effectively mean that the computation of a particular estimated cycle will be extremely expensive (as extremely fine discretisation is needed to capture the micro-cycles). Employing cycle jump is not theoretically possible for simulating micro-cycles, especially if the macroscopic variation is highly non-linear (which is indeed the case for $\mathrm{CCF}$ ) as cycles need to be periodic or at least periodic by block (which does not exist for CCF type loads). Therefore the best strategy is to adopt a combined technique, taking advantages of both while minimising their frailties. As far as the engineering components of interest is concerned, one can always think of turbine blades of gas turbines (where anisothermal conditions exist along with high pressure mechanical loads) in jet engines as an example. Internal 
combustion engines (especially the connecting rod, and the crankshaft) also operate under such an extreme condition and is also subjected to thermo-mechanical fatigue.

The authors would like herein to emphasise the fact that the content of this article merely focuses on numerical approach, and is provided as a tool for structural analysis for the fatigue and damage community.

The articles begins by introducing the reference thermo-mechanical problem in section 2 . The temporal homogenisation procedure is detailed in section 3 including scale separations for both the thermal and the mechanical problem, for a particular macro-cycle. Thereafter, the classical cycle jump method is described in section 4 along with the overall computational procedure. Finally a numerical case study is presented in section 5 along with some verification analyses.

\section{The reference problem}

Considering the thermal problem as depicted in fig. 1a, Fourier's law of heat conduction reads

$$
\underline{q}=-k_{t} \nabla \theta, \text { in } \Omega,
$$

where $q$ is the heat flux vector, $\theta$ is the temperature field and $\Omega$ is the spatial domain of the reference problem. The thermal conductivity $k_{t}$ is considered to be isotropic. The heat flow equation at a given time $t$, in the absence of internal heat source reads

$$
-\nabla \cdot \underline{q}=\rho c_{t} \frac{\mathrm{d} \theta}{\mathrm{d} t}, \text { in } \Omega,
$$

where $\rho$ is the density of the material, and $c_{t}$ is the specific heat capacity. Equations (1) and (2) can be combined to provide the complete transient heat equation within the domain $\Omega$ as

$$
k_{t} \nabla^{2} \theta=\rho c_{t} \frac{\mathrm{d} \theta}{\mathrm{d} t} .
$$

For simplicity, it is considered that there is no adiabatic overheating, i.e. there is no internal heat generation due to mechanical dissipation or any other sources, i.e. the thermo-mechanical coupling is one way.

The boundary conditions of the structure can be of Dirichlet type i.e.

$$
\theta=\theta_{d}, \text { on } \partial \Omega_{t 1},
$$

where $\theta_{d}$ is the prescribed temperature on the boundary $\partial \Omega_{t 1}$. The Neumann type boundary condition on the complementary surface can be written as

$$
\underline{q} \cdot \underline{n}=-q_{s}, \text { on } \partial \Omega_{t 2},
$$

where $\underline{n}$ is the unit vector normal to the surface $\partial \Omega_{t 2}, q_{s}$ is the heat flux across the boundary from external sources.

The resolution of eq. (3) along with eqs. (4) and (5) provides the temperature field $\theta$ in the whole structure, which can be used to calculate the thermal strain $\varepsilon^{\text {th }}$ at each material point as,

$$
\varepsilon^{t h}=\varpi\left(\theta-\theta_{i n}\right) \delta .
$$

The material parameter $\varpi$ is the coefficient of linear thermal expansion, $\theta_{i n}$ is the initial temperature (at the start of loading), and $\delta$ is identity tensor. The thermal strain hence calculated is considered as an input in the mechanical problem. 


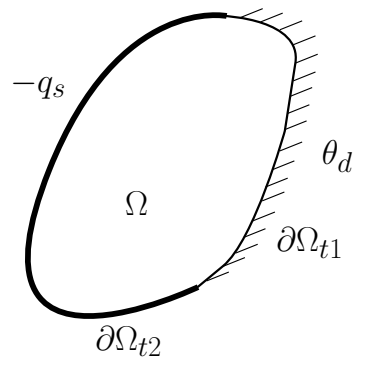

(a) Thermal problem

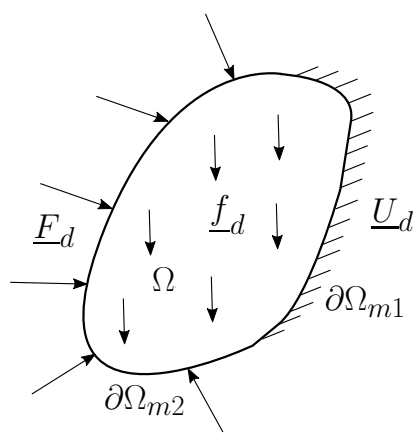

(b) Mechanical problem

Figure 1: Reference problem in domain $\Omega$

Considering now the mechanical reference problem in the domain $\Omega$ (fig. $1 \mathrm{~b}$ ), subjected to body force $f_{d}$. The boundary $\partial \Omega$ of the domain is sundered into $\partial \Omega_{m 1}$ where a prescribed displacement $\underline{U}_{d}$ is specified, and $\partial \Omega_{m 2}$ containing applied surface traction $\underline{F}_{d}$. The admissibility conditions that dictate such a problem is given as

- Static admissibility condition

$$
\begin{aligned}
& \nabla \cdot \boldsymbol{\sigma}+\underline{f}_{d}=0, \text { in } \Omega, \\
& \underline{F}_{d}=\boldsymbol{\sigma} \cdot \underline{n}, \text { on } \partial \Omega_{m 2} .
\end{aligned}
$$

The quantity $\boldsymbol{\sigma}$ represents the Cauchy stress tensor and $\underline{n}$ is the unit normal at each point on the surface. The case considered here is a quasi-static problem hence the inertia term is neglected.

- Kinematic admissibility condition

$$
\begin{aligned}
& \varepsilon=\frac{1}{2}\left[\nabla \underline{u}+(\nabla \underline{u})^{T}\right], \text { in } \Omega, \\
& \underline{u}=\underline{U}_{d}, \text { on } \partial \Omega_{m 1},
\end{aligned}
$$

where $\varepsilon$ is the infinitesimal strain tensor and $\underline{u}$ is the displacement field.

Apart from the global admissibility conditions, local constitutive relations are also required at each material point, to have a closed form mechanical problem. In the current article, the material behaviour considered is thermo-elasto-viscoplasticity with mixed hardening coupled with damage (see [23, 24] for details). The considered constitutive relations are

- State laws

$$
\begin{aligned}
\boldsymbol{\sigma} & =\mathbf{C}(1-D)\left(\varepsilon^{e}-\varepsilon^{t h}\right) \\
\boldsymbol{\beta} & =\mathbf{Q} \boldsymbol{\alpha} \\
R & =R_{\infty}(1-\exp (-\gamma r)) \\
Y & =\mathcal{G}(\boldsymbol{\sigma}, D), \text { with } \mathcal{G}=\frac{\tilde{\sigma}_{e q}^{2} R_{v}}{2 E} .
\end{aligned}
$$


$\mathbf{C}$ is the Hooke's tensor which is a function the modulus of elasticity $E$ and Poisson's ratio $\nu$. $\mathbf{Q}$ is a tensor containing material parameter $Q$ that describes kinematic hardening. $R_{\infty}$ and $\gamma$ are material parameters describing isotropic hardening. $\varepsilon^{e}$ is the elastic part of the total strain such that

$$
\varepsilon=\varepsilon^{e}+\varepsilon^{p}
$$

where $\varepsilon^{p}$ is the inelastic or plastic strain. $\varepsilon^{t h}$ is the thermal strain obtained from the resolution of the thermal problem. $\boldsymbol{\alpha}$ and $\boldsymbol{\beta}$ are the internal variable and its conjugate respectively, describing the linear kinematic hardening. $r$ and $R$ are similar internal and conjugate variable respectively, describing the exponential isotropic hardening. The quantity $Y$ represents the strain energy release rate associated with the isotropic damage variable $(D \in[0,1])$. The triaxiality function $R_{v}=\frac{2}{3}(1+\nu)+3(1-2 \nu)\left(\frac{\sigma_{h}}{\sigma_{e q}}\right)^{2}$, where $\sigma_{h}=\frac{1}{3} \operatorname{Tr}(\boldsymbol{\sigma})$ represents the hydrostatic part of the stress tensor, $\sigma_{e q}$ is the von Mises equivalent stress defined as $\sigma_{e q}=\sqrt{\frac{3}{2} \boldsymbol{\sigma}^{D}: \boldsymbol{\sigma}^{D}}$ with $\boldsymbol{\sigma}^{D}=\boldsymbol{\sigma}-\sigma_{h} \delta$ being the deviatoric stress. The ratio $\frac{\sigma_{h}}{\sigma_{e q}}$ in the definition of $R_{v}$ is called the triaxiality ratio and $\tilde{\sigma}_{e q}$ is the equivalent of the effective stress defined as $\tilde{\sigma}_{e q}=\sqrt{\frac{3}{2} \frac{\boldsymbol{\sigma}^{D}}{(1-D)}: \frac{\boldsymbol{\sigma}^{D}}{(1-D)}}$.

- Evolution equations

$$
\begin{aligned}
& \frac{\mathrm{d} \varepsilon^{p}}{\mathrm{~d} t}=\frac{\partial f^{p}}{\partial \boldsymbol{\sigma}} \dot{\lambda}, \\
& \frac{\mathrm{d} \boldsymbol{\alpha}}{\mathrm{d} t}=-\frac{\partial f^{p}}{\partial \boldsymbol{\beta}} \dot{\lambda}, \\
& \frac{\mathrm{d} r}{\mathrm{~d} t}=-\frac{\partial f^{p}}{\partial R} \dot{\lambda}, \\
& \frac{\mathrm{d} D}{\mathrm{~d} t}=\left(\frac{Y}{S}\right)^{s} \frac{\dot{\lambda}}{1-D},
\end{aligned}
$$

with the von Mises yield function $f^{p}$ delimiting the elastic domain and is defined as

$$
f^{p}=\sqrt{\frac{3}{2}\left[\left(\frac{\boldsymbol{\sigma}^{D}}{1-D}-\boldsymbol{\beta}\right):\left(\frac{\boldsymbol{\sigma}^{D}}{1-D}-\boldsymbol{\beta}\right)\right]}-R-\sigma_{y}
$$

where $\sigma_{y}$ is the yield stress. The viscoplastic multiplier $\dot{\lambda}$ defined according to Norton's law as,

$$
\dot{\lambda}=k_{v}\left\langle f^{p}\right\rangle^{n_{v}}
$$

where the viscous coefficient $k_{v}$ and viscous exponent $n_{v}$ are material parameters. The material parameters $S$ and $s$ describe the damage evolution.

The constitutive relations and the admissibility conditions enable the resolution of the mechanical problem with the primary quantity of interest being the damage variable $D$. For the sake of later developments, eq. 11$\}$ is represented as $\frac{\mathrm{d} \varphi}{\mathrm{d} t}=\mathcal{M}(\psi)$, where $\varphi=\left\{\varepsilon^{p}, \boldsymbol{\alpha}, r, D\right\}$ and $\psi=\{\boldsymbol{\sigma}, \boldsymbol{\beta}, R, D\}$. 
The prescribed loading, i.e. $q_{s}, \theta_{d}, \underline{u}_{d}, \underline{f}_{d}$, and $\underline{F}_{d}$ are of CCF type, with large number of macro cycles and each of them containing a large number of micro cycles. Figure 2 shows a generic CCF loading for a prescribed load $\chi$, where the total number of load cycles is the product of the number of macro cycles and the number of micro cycles for each macro cycle. Classical cycle-by-cycle simulation in such cases is extremely costly at both macro and micro scale. In the next section, the technique for the resolution of one macro cycle is detailed.

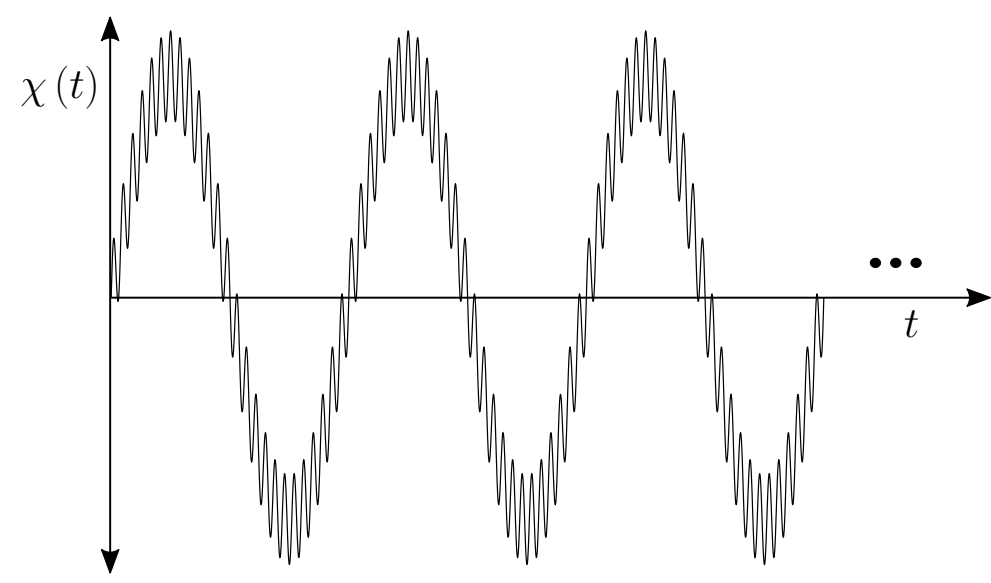

Figure 2: Considered CCF type loading

\section{Temporal homogenisation for a given macro cycle}

The details regarding classical temporal homogenisation can be found in 3, 30, 31. The application with respect combined cycle fatigue damage is summarised herein, along with the derivation for the thermal case.

The main philosophy is based on the existence of two separate scales

- A slow time scale $t$ associated with the macro cycles,

- A fast time scale $\tau$ associated with the micro cycles.

This thereby allows to define a time scale ratio

$$
\xi=\frac{t}{\tau}=\frac{\mathcal{T}_{m}}{\mathcal{T}_{M}},
$$

where $\mathcal{T}_{m}$ and $\mathcal{T}_{M}$ are the time periods of the micro and the macro cycles, respectively. It is obvious that $0<\xi \ll 1$, and smaller the value of $\xi$, better is the decoupling of the two scales. Considering $\xi$ to be infinitesimal, any time dependent quantity becomes function of both $t$ and $\tau$. The time derivative of such a quantity $\chi(t, \tau)$ can be written as

$$
\frac{\mathrm{d} \chi}{\mathrm{d} t}=\frac{\partial \chi}{\partial t}+\frac{1}{\xi} \frac{\partial \chi}{\partial \tau}=\dot{\chi}+\frac{\chi^{\prime}}{\xi}
$$

It is further assumed that the quantity $\chi(t, \tau)$ is quasi-periodic or locally periodic with respect to $\tau$, i.e.

$$
\chi(t, \tau)=\chi\left(t, \tau+\mathcal{T}_{m}\right) .
$$


The quasi-periodicity assumption also means that

$$
\left\langle\chi^{\prime}\right\rangle_{\tau}=0,
$$

where $\langle 0\rangle_{\tau}$ represents the averaging operator relative to $\tau$ such that

$$
\langle\chi\rangle_{\tau}(t)=\frac{1}{\mathcal{T}_{m}} \int_{0}^{\mathcal{T}_{m}} \chi(t, \tau) \mathrm{d} \tau,
$$

where $\langle\chi\rangle_{\tau}$ is the averaged or homogenised quantity and is a function of $t$ only. Such a homogenisation also allows to define a residual quantity $\chi^{*}(t, \tau)$, such that

$$
\chi=\chi^{*}+\langle\chi\rangle_{\tau} .
$$

\subsection{Scale separation for the thermal problem}

The thermal problem due to its linearity can be decoupled in the two scales based on classical superposition principle.

Expressing the load $q_{s}(t, \tau)$ and $\theta_{d}(t, \tau)$ being of the nature as in eq. (19), eqs. (3) to (5) can be re-written as a residual problem (with $\theta^{*}$ being $\tau$ periodic),

$$
\begin{aligned}
& k_{t} \nabla^{2} \theta^{*}=\rho c_{t} \frac{\mathrm{d} \theta^{*}}{\mathrm{~d} \tau} \operatorname{in} \Omega, \\
& \theta^{*}=\theta_{d}^{*}, \text { on } \partial \Omega_{t 1}, \\
& \underline{q}^{*} \cdot \underline{n}=-q_{s}^{*}, \text { on } \partial \Omega_{t 2},
\end{aligned}
$$

and as a homogenised problem,

$$
\begin{aligned}
& k_{t} \nabla^{2}\langle\theta\rangle_{\tau}=\rho c_{t} \frac{\mathrm{d}\langle\theta\rangle_{\tau}}{\mathrm{d} t} \text { in } \Omega, \\
& \langle\theta\rangle_{\tau}=\left\langle\theta_{d}\right\rangle_{\tau}, \text { on } \partial \Omega_{t 1}, \\
& \langle\underline{q}\rangle_{\tau} \cdot \underline{n}=-\left\langle q_{s}\right\rangle_{\tau}, \text { on } \partial \Omega_{t 2} .
\end{aligned}
$$

The resolution of which will provide the residual field $\theta^{*}$ and the homogenised field $\langle\theta\rangle_{\tau}$ respectively.

\subsection{Scale separation for the mechanical problem}

The non-linearity of the mechanical problem prevents the application of the superposition principle. Therefore any quantity $\chi$ from the mechanical problem is written as an asymptotic expansion with respect to the time scale ratio $\xi$, i.e.

$$
\chi(t, \tau)=\chi_{0}(t, \tau)+\xi \chi_{1}(t, \tau)+\xi^{2} \chi_{2}(t, \tau)+\cdots .
$$

Lower the value of $\xi$, better the approximation. Such expanded form is used to re-write all the governing equations of the problem at hand.

Expressing the loads $\underline{U}_{d}, f_{d}$ and $\underline{F}_{d}$ as in eq. $(19)$, and applying the asymptotic development (eq. (22)), on eqs. (7) to (9) and eq. (10), the admissibility conditions and the state laws can be 


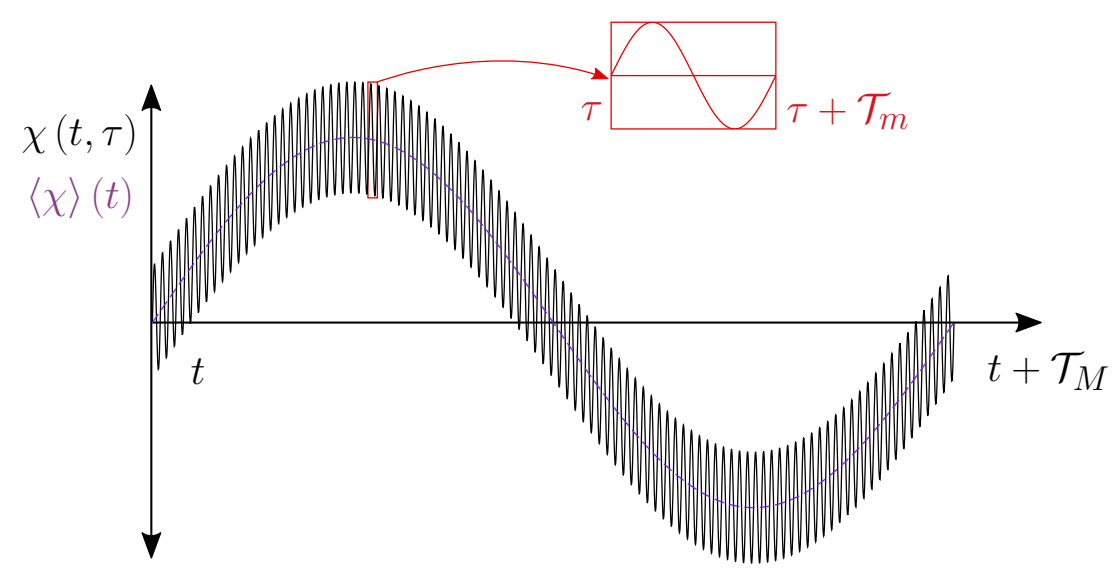


written as

$$
\begin{aligned}
& \nabla \cdot\left(\boldsymbol{\sigma}_{0}+\xi \boldsymbol{\sigma}_{1}+\cdots\right)+\underline{f}_{d}=0, \text { in } \Omega, \\
& \underline{F}_{d}=\left(\boldsymbol{\sigma}_{0}+\xi \boldsymbol{\sigma}_{1}+\cdots\right) \cdot \underline{n}, \text { on } \partial \Omega_{m 2}, \\
& \varepsilon_{0}+\xi \varepsilon_{1}+\cdots=\frac{1}{2}\left[\nabla\left(\underline{u}_{0}+\xi \underline{u}_{1}+\cdots\right)+\left(\nabla\left(\underline{u}_{0}+\xi \underline{u}_{1}+\cdots\right)\right)^{T}\right], \text { in } \Omega, \\
& \underline{u}_{0}+\xi \underline{u}_{1}+\cdots=\underline{U}_{d}, \text { on } \partial \Omega_{m 1}, \\
& \boldsymbol{\sigma}_{0}+\xi \boldsymbol{\sigma}_{1}+\cdots=\mathbf{C}\left(1-\left(D_{0}+\xi D_{1}+\cdots\right)\right)\left(\left(\varepsilon_{0}^{e}+\xi \varepsilon_{1}^{e}+\cdots\right)-\varepsilon^{t h}\right) \\
& \boldsymbol{\beta}_{0}+\xi \boldsymbol{\beta}_{1}+\cdots=\mathbf{Q}\left(\boldsymbol{\alpha}_{0}+\xi \boldsymbol{\alpha}_{1}+\cdots\right), \\
& R_{0}+\xi R_{1}+\cdots=R_{\infty}\left(1-\exp \left(-\gamma\left(r_{0}+\xi r_{1}+\cdots\right)\right)\right), \\
& Y_{0}+\xi Y_{1}+\cdots=\mathcal{G}\left(\boldsymbol{\sigma}_{0}+\xi \boldsymbol{\sigma}_{1}+\cdots, D_{0}+\xi D_{1}+\cdots\right), \\
& \varepsilon_{0}+\xi \varepsilon_{1}+\cdots=\left(\varepsilon_{0}^{e}+\xi \varepsilon_{1}^{e}+\cdots\right)+\left(\varepsilon_{0}^{p}+\xi \varepsilon_{1}^{p}+\cdots\right),
\end{aligned}
$$

Using eqs. (15) and (22), eq. (11) can be rewritten as

$$
\begin{array}{r}
\frac{\partial}{\partial t}\left(\varphi_{0}+\xi \varphi_{1}+\cdots\right)+\frac{1}{\xi} \frac{\partial}{\partial \tau}\left(\varphi_{0}+\xi \varphi_{1}+\cdots\right)=\mathcal{M}\left(\psi_{0}+\xi \psi_{1}+\cdots\right) \\
=\mathcal{M}\left(\psi_{0}\right)+\left.\xi \frac{\partial \mathcal{M}}{\partial \psi}\right|_{\psi_{0}} \psi_{1}+\cdots
\end{array}
$$

The idea hereafter is to equate terms having the same power of $\xi$ for eq. (23) and eq. (24) to generate different order of problems.

-1 order problem Equating the terms having $1 / \xi$ in eq. (24),

$$
\frac{\partial \varphi_{0}}{\partial \tau}=0 \Longrightarrow \varphi_{0}=\varphi_{0}(t),
$$

Thereby from eqs. 23f] and (23g) it is evident that $\boldsymbol{\beta}_{0}=\boldsymbol{\beta}_{0}(t)$ and $R_{0}=R_{0}(t)$.

This basically means that the zero order terms of $\varepsilon^{p}, \boldsymbol{\alpha}, \boldsymbol{\beta}, r, R$ and $D$ are dependent on the macroscopic time only.

0 order problem Now, equating terms without $\xi$ in eq. (23) and eq. (24),

$$
\begin{aligned}
& \nabla \cdot \boldsymbol{\sigma}_{0}+\underline{f}_{d}=0, \text { in } \Omega, \\
& \underline{F}_{d}=\boldsymbol{\sigma}_{0} \cdot \underline{n}, \text { on } \partial \Omega_{m 2}, \\
& \varepsilon_{0}=\frac{1}{2}\left[\nabla \underline{u}_{0}+\left(\nabla \underline{u}_{0}\right)^{T}\right], \text { in } \Omega, \\
& \underline{u}_{0}=\underline{U}_{d}, \text { on } \partial \Omega_{m 1}, \\
& \boldsymbol{\sigma}_{0}=\mathbf{C}\left(1-D_{0}\right)\left(\varepsilon_{0}^{e}-\boldsymbol{\varepsilon}^{t h}\right) \\
& \boldsymbol{\beta}_{0}=\mathbf{Q} \boldsymbol{\alpha}_{0}, \\
& R_{0}=R_{\infty}\left(1-\exp \left(-\gamma r_{0}\right)\right), \\
& Y_{0}=\mathcal{G}\left(\boldsymbol{\sigma}_{0}, D_{0}\right), \\
& \varepsilon_{0}=\varepsilon_{0}^{e}+\varepsilon_{0}^{p} . \\
& \frac{\partial \varphi_{0}}{\partial t}+\frac{\partial \varphi_{1}}{\partial \tau}=\mathcal{M}\left(\psi_{0}\right),
\end{aligned}
$$


Using the average operator defined in eq. (18), the quasi-periodicity assumption of eq. (17), and eq. (19), eq. 26) becomes

$$
\begin{aligned}
& \nabla \cdot\left\langle\boldsymbol{\sigma}_{0}\right\rangle_{\tau}(t)+\left\langle\underline{f}_{d}\right\rangle_{\tau}(t)=0, \text { in } \Omega, \\
& \left\langle\underline{F}_{d}\right\rangle_{\tau}(t)=\left\langle\boldsymbol{\sigma}_{0}\right\rangle_{\tau}(t) \cdot \underline{n}, \text { on } \partial \Omega_{m 2}, \\
& \left\langle\varepsilon_{0}\right\rangle_{\tau}(t)=\frac{1}{2}\left[\nabla\left\langle\underline{u}_{0}\right\rangle_{\tau}(t)+\left(\nabla\left\langle\underline{u}_{0}\right\rangle_{\tau}(t)\right)^{T}\right], \text { in } \Omega, \\
& \left\langle\underline{u}_{0}\right\rangle_{\tau}(t)=\left\langle\underline{U}_{d}\right\rangle_{\tau}(t), \text { on } \partial \Omega_{m 1}, \\
& \left\langle\boldsymbol{\sigma}_{0}\right\rangle_{\tau}(t)=\mathbf{C}\left(1-D_{0}(t)\right)\left(\left\langle\varepsilon_{0}^{e}\right\rangle_{\tau}(t)-\left\langle\varepsilon^{t h}\right\rangle_{\tau}(t)\right) \\
& \boldsymbol{\beta}_{0}(t)=\mathbf{Q} \boldsymbol{\alpha}_{0}(t), \\
& R_{0}(t)=R_{\infty}\left(1-\exp \left(-\gamma r_{0}(t)\right)\right), \\
& \left\langle Y_{0}\right\rangle_{\tau}(t)=\left\langle\mathcal{G}\left(\boldsymbol{\sigma}_{0}^{*}+\left\langle\boldsymbol{\sigma}_{0}\right\rangle_{\tau}, D_{0}\right)\right\rangle_{\tau}(t), \\
& \left\langle\varepsilon_{0}\right\rangle_{\tau}(t)=\left\langle\varepsilon_{0}^{e}\right\rangle_{\tau}(t)+\varepsilon_{0}^{p}(t) . \\
& \frac{\partial \varphi_{0}}{\partial t}=\left\langle\mathcal{M}\left(\psi_{0}^{*}+\left\langle\psi_{0}\right\rangle_{\tau}\right)\right\rangle_{\tau}=\left\langle\mathcal{M}\left(\boldsymbol{\sigma}_{0}^{*}+\left\langle\boldsymbol{\sigma}_{0}\right\rangle_{\tau}, \boldsymbol{\beta}_{0}, R_{0}, D_{0}\right)\right\rangle_{\tau},
\end{aligned}
$$

It has to be noted that due to the outcome of the -1 order problem, $\left\langle\boldsymbol{\beta}_{0}\right\rangle_{\tau}=\boldsymbol{\beta}_{0},\left\langle\boldsymbol{\alpha}_{0}\right\rangle_{\tau}=\boldsymbol{\alpha}_{0}$, $\left\langle r_{0}\right\rangle_{\tau}=r_{0},\left\langle R_{0}\right\rangle_{\tau}=R_{0},\left\langle D_{0}\right\rangle_{\tau}=D_{0}$ and $\left\langle\varepsilon_{0}^{p}\right\rangle_{\tau}=\varepsilon_{0}^{p}$. Equation 27$]$ is the zero order homogenised problem with prescribed macroscopic mechanical loads $\left\langle\underline{f}_{d}\right\rangle_{\tau},\left\langle\underline{F}_{d}\right\rangle_{\tau}$ and $\left\langle\underline{U}_{d}\right\rangle_{\tau}$. The input from the thermal problem is through the homogenised thermal strain which is given as,

$$
\left\langle\varepsilon^{t h}\right\rangle_{\tau}=\varpi\left(\langle\theta\rangle_{\tau}-\theta_{i n}\right) \delta .
$$

It has to be mentioned that the solution of eq. (27) requires the knowledge of the residual stress field $\boldsymbol{\sigma}_{0}^{*}$. Subtracting eq. (27) from eq. 26] to obtain the zero order residual problem,

$$
\begin{aligned}
& \nabla \cdot \boldsymbol{\sigma}_{0}^{*}+\underline{f}_{d}^{*}=0, \text { in } \Omega, \\
& \underline{F}_{d}^{*}=\boldsymbol{\sigma}_{0}^{*} \cdot \underline{n}, \text { on } \partial \Omega_{m 2}, \\
& \varepsilon_{0}^{*}=\frac{1}{2}\left[\nabla \underline{u}_{0}^{*}+\left(\nabla \underline{u}_{0}^{*}\right)^{T}\right], \text { in } \Omega, \\
& \underline{u}_{0}^{*}=\underline{U}_{d}^{*}, \text { on } \partial \Omega_{m 1}, \\
& \boldsymbol{\sigma}_{0}^{*}=\mathbf{C}\left(1-D_{0}\right)\left(\varepsilon_{0}^{e *}-\varepsilon^{t h *}\right) \\
& \varepsilon_{0}^{*}=\varepsilon_{0}^{e *},
\end{aligned}
$$

which is indeed a thermo-elastic problem parametrised by the zero order damage variable $D_{0}$. The quntities $\underline{f}_{d}^{*}, \underline{F}_{d}^{*}$ and $\underline{U}_{d}^{*}$ are the prescribed microscopic loads and the residual thermal strain is obtained as

$$
\varepsilon^{t h *}=\varpi\left(\theta^{*}-\theta_{i n}\right) \delta
$$

The resolution of eq. 29 will provide the residual stress field $\boldsymbol{\sigma}_{0}^{*}$ (along with $\varepsilon_{0}^{*}$ and $\underline{u}_{0}^{*}$ ) which can be used to solve the homogenised problem.

Of course it is possible to obtain higher order equations (see e.g. [31]), however in this particular case the problem is restricted to zero order approximation. 


\section{Cycle jump method in the overall scheme}

Although, the philosophy of homogenisation reduces the numerical cost as it avoids cycle-by-cycle simulation of the micro-cycles, the existence of large number of macro cycles increase the overall numerical expense. Hence, a classical cycle jump algorithm (as described in [24]) is employed to avoid cycle-by-cycle simulation of the macro-cycles.

The main problem of cycle jump is to obtain the initial condition for a macro-cycle of interest. The residual thermal problem (eq. (20) ) is computed using zero initial condition once for all, thereby the resolution of eq. (20) will not depend on the macro-initial condition. The residual mechanical problem (eq. $(29)$ ) is a linear problem in $\tau$, however requiring the knowledge of the macroscopic damage variable $D_{0}$. For the homogenised problems (eqs. (21) and (27)) also the information of the initial conditions are necessary to solve ODEs in $t$.

Considering cycle number $N_{s}$ has been simulated, the next $\Delta N_{s}$ cycles to be jumped is given by

$$
\Delta N_{s}=\frac{D_{c} / 50}{\left.\frac{\mathrm{d} D_{0}}{\mathrm{~d} N}\right|_{N_{s}}}
$$

where $D_{c}$ is the critical damage. The factor of 50 has been chosen in accordance with 25. It essentially controls the number of cycles to be jumped and can be altered according to desired numerical accuracy or computational cost of the approach.

The next macro-cycle to be simulated is given by

$$
N_{s+1}=N_{s}+\Delta N_{s}+1
$$

For all quantities which are cyclic, the final value at $N_{s}$ is considered as the initial value at $N_{s+1}$, i.e. for a cyclic quantity $\chi=\left\{\langle\theta\rangle_{\tau},\left\langle u_{0}\right\rangle_{\tau},\left\langle\boldsymbol{\sigma}_{0}\right\rangle_{\tau},\left\langle\varepsilon_{0}\right\rangle_{\tau},\left\langle\varepsilon_{0}^{e}\right\rangle_{\tau}, \boldsymbol{\varepsilon}_{0}^{p}, \boldsymbol{\alpha}_{0}, \boldsymbol{\beta}_{0}\right\}$,

$$
\chi_{i n}\left(N_{s+1}\right)=\chi_{f i}\left(N_{s}\right),
$$

where $\chi_{i n}$ and $\chi_{f i}$ represent the initial and final value at the respective cycles $N_{s+1}$ and $N_{s}$. For quantities that are not cyclic, namely $D_{0}, R_{0}, r_{0}$, linear extrapolations of the form

$$
\begin{aligned}
& D_{0, i n}\left(N_{s+1}\right)=D_{0, f i}\left(N_{s}\right)+\left.\frac{\mathrm{d} D_{0}}{\mathrm{~d} N}\right|_{N_{s}} \Delta N_{s}, \\
& R_{0, i n}\left(N_{s+1}\right)=R_{0, f i}\left(N_{s}\right)+\left.\frac{\mathrm{d} R_{0}}{\mathrm{~d} N}\right|_{N_{s}} \Delta N_{s}, \\
& r_{0, i n}\left(N_{s+1}\right)=r_{0, f i}\left(N_{s}\right)+\left.\frac{\mathrm{d} r_{0}}{\mathrm{~d} N}\right|_{N_{s}} \Delta N_{s},
\end{aligned}
$$

are used. The initial value of the strain energy release rate if needed can be calculated from eq. (27h).

The presence of viscoplasticity, renders the initial macro cycles to be non-periodic. Hence, initial few macro cycles are calculated without any jumps. After that, the cycle jump technique is employed. For a cycle of interest $N_{s}$, with the given initial conditions at $t_{N_{s}}$, the residual mechanical problem (eq. (29)) is solved for $\tau \in\left[0, \mathcal{T}_{m}\right]$. Thereafter at each macro time step $t \in\left[t_{N_{s}}, t_{N_{s}}+\mathcal{T}_{M}\right]$, a classical elastic predictor-plastic corrector method is applied on a global Newton-Raphson algorithm. It should be noted that in the plastic corrector step the right-handsides of eq. 27j] are averages of all the micro time steps. The solution of this non-linear material behaviour can be achieved by using any non-linear solver (like Newton-Raphson methods or 
trust-region algorithms). At each iteration of the global Newton Raphson algorithm, eq. (29) needs to be solved with the new value of $D_{0}$ to update $\boldsymbol{\sigma}_{0}^{*}, \varepsilon_{0}^{*}$ and $\underline{u}_{0}^{*}$. The residual thermal problem needs to be solved only once for $\tau \in\left[0, \mathcal{T}_{m}\right]$, and the homogenised thermal problem is solved at each macro time step before the Newton-Raphson algorithm. Once $t=t_{N_{s}}+\mathcal{T}_{M}$ is reached, the new $N_{s+1}$ is calculated and the whole procedure is repeated. This continues till the maximum damage value $D_{0}$ reaches the critical value $D_{c}$. Once all the macro cycles are calculated, they can be post-treated to obtain the complete evolution of the desired quantities of interest using linear interpolation functions (see [7, 11] for details).

\section{Numerical study}

The structure considered for the numerical study is a rectangular block with a circular groove (see fig. 4). The dimension of the structure is given by $L=W=100 \mathrm{~mm}, H=20 \mathrm{~mm}$ and the radius of the groove $\varrho=20 \mathrm{~mm}$. The block is subjected to uniform traction $F_{d}=\left\langle F_{d}\right\rangle_{\tau}+F_{d}^{*}$, with $\left\langle F_{d}\right\rangle_{\tau}=7 \sin \left(\frac{2 \pi t}{\mathcal{T}_{M}}\right) \mathrm{N} / \mathrm{mm}^{2}$ and $F_{d}^{*}=1.5 \sin \left(\frac{2 \pi \tau}{\mathcal{T}_{m}}\right) \mathrm{N} / \mathrm{mm}^{2}$ on the top and bottom surface of the block. The time periods of the loads are given by $\mathcal{T}_{M}=20000 \mathrm{~s}$ and $\mathcal{T}_{m}=20$ s, i.e. $\xi=10^{-3}$. The block is also subjected to a temperature field of the form $\theta_{d}=\left\langle\theta_{d}\right\rangle_{\tau}+\theta_{d}^{*}$ with $\left\langle\theta_{d}\right\rangle_{\tau}=40 \sin \left(\frac{2 \pi t}{\mathcal{T}_{M}}\right) \cos \left(\frac{\pi z}{H}\right){ }^{\circ} \mathrm{C}$ and $\theta_{d}^{*}=10 \sin \left(\frac{2 \pi \tau}{\mathcal{T}_{m}}\right) \cos \left(\frac{\pi z}{H}\right){ }^{\circ} \mathrm{C}$, on the inner surface of the cylindrical groove, i.e they are maximum on the symmetric surface $(z=0)$ and 0 on the external surfaces $(z= \pm H / 2)$. Temperatures on the six outer faces are fixed at a constant value $\theta_{f}=0{ }^{\circ} \mathrm{C}$. Due to symmetry, only one-eighth of the structure is used for the analysis (see fig. (4). It is probably noteworthy to mention that the choice of such a problem is purely arbitrary, and any other academic problem would also have sufficed.

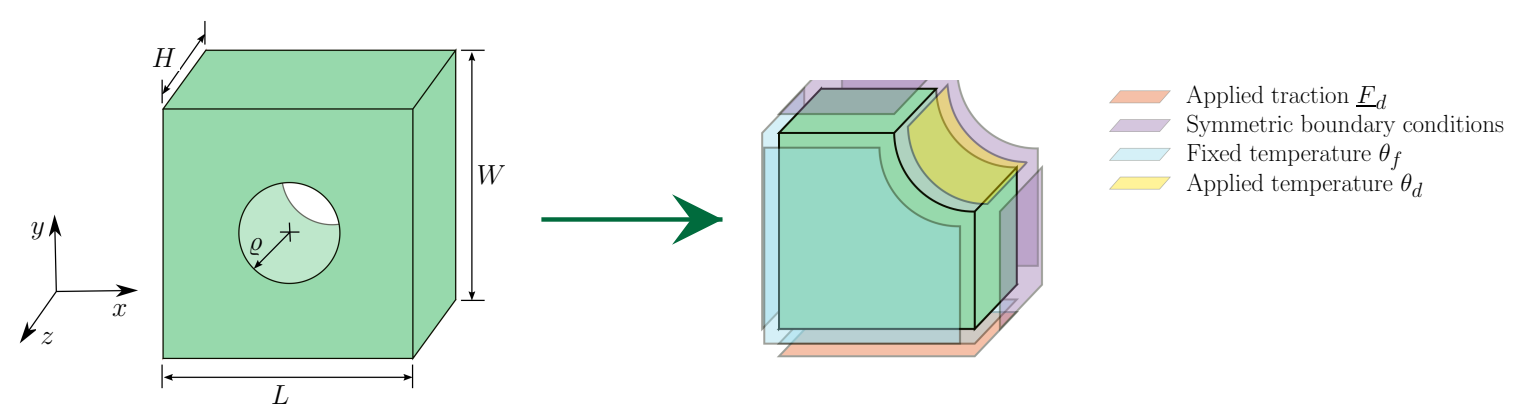

Figure 4: Structure under thermo-mechanical CCF

The spatial discretisation is achieved by using 940 8-noded linear isoparametric hexahedral elements with 8 Gauss points per element, i.e. a total of 7520 Gauss points and 1345 nodes.

The material used is a Cr-Mo Steel, the approximate material parameters of which is given in table 1 .

\subsection{Verification analyses}

To exemplify the accuracy of the procedure, a few test analyses are performed. The first is to check the accuracy of the temporal homogenisation, for which only one macro cycle is considered 


\begin{tabular}{cccccccc}
\hline$E$ & $\nu$ & $\sigma_{y}$ & $Q$ & $R_{\infty}$ & $\gamma$ & $K_{v}$ & $n_{v}$ \\
$134000 \mathrm{MPa}$ & 0.3 & $85 \mathrm{MPa}$ & $6000 \mathrm{MPa}$ & $30 \mathrm{MPa}$ & 2 & $1220 \mathrm{MPa} \mathrm{s}^{1 / n_{v}}$ & 2.5 \\
\hline \hline$k_{v}$ & $S$ & $s$ & $D_{c}$ & $k_{t}$ & $\rho c_{t}$ & $\varpi$ \\
$K_{v}^{-n_{v}}$ & $0.6 \mathrm{MPa}$ & 2 & 0.2 & $38 \mathrm{~J} / \mathrm{m}^{3} /{ }^{\circ} \mathrm{C}$ & $5.46 \mathrm{~W} / \mathrm{m}^{3} /{ }^{\circ} \mathrm{C}$ & $1.3 \times 10^{-5} 1 /{ }^{\circ} \mathrm{C}$ \\
\hline
\end{tabular}

Table 1: Material parameters

and the homogenised solution is compared with a reference solution obtained using 30000 time steps, i.e. 30 time steps per micro cycle. For the two scale solution, 30 time steps are considered for the micro cycle and 60 time steps for the macro cycle. The accuracy is measured by an error criteria

$$
e r=\left(\frac{\int_{\Omega \times[0, T]}\left(D_{r e f}-D_{t s}\right) \cdot\left(D_{r e f}-D_{t s}\right) \mathrm{d} \Omega \mathrm{d} t}{\int_{\Omega \times[0, T]}\left(D_{r e f}+D_{t s}\right) \cdot\left(D_{r e f}+D_{t s}\right) \mathrm{d} \Omega \mathrm{d} t}\right)^{1 / 2},
$$

with $T$ being the total temporal domain, $D_{r e f}$ is the reference solution at the macroscopic time points, and $D_{t s}$ the homogenised solution. An error of $3 \%$ is achieved with CPU time gain of $95 \%$, proving that there is a tremendous gain in CPU time at an acceptable accuracy.

The second test is to check the accuracy of the jump cycle method, for which only the macroscopic loads $\left\langle\underline{F}_{d}\right\rangle_{\tau}$ and $\left\langle\theta_{d}\right\rangle_{\tau}$ are considered, i.e. the problem is a mono-scale problem. These loads are applied for 1000 cycles and cycle jump method is compared with a reference cycle-by-cycle simulation. 30 time steps per cycle is considered for both the simulations, with a fixed jumping of 100 cycles to avoid over jump (since the total number of considered cycles is low). The effect of the number of jumped cycles on the accuracy for cycle jump methods has been investigated in [7] rigorously, here just a test case is investigated. For the simulation using the cycle jump technique, the first 3 cycles are calculated directly, thereafter jumping is applied, resulting in the effective simulation of 13 cycles out of 1000 . An error criteria defined as

$$
e r=\left(\frac{\int_{\Omega \times[0, T]}\left(D_{r e f}-D_{j u m}\right) \cdot\left(D_{r e f}-D_{j u m}\right) \mathrm{d} \Omega \mathrm{d} t}{\int_{\Omega \times[0, T]}\left(D_{r e f}+D_{j u m}\right) \cdot\left(D_{r e f}+D_{j u m}\right) \mathrm{d} \Omega \mathrm{d} t}\right)^{1 / 2}
$$

with $T$ being the total temporal domain, $D_{r e f}$ is the reference solution, and $D_{j u m}$ is the interpolated solution $\forall t \in[0, T]$. The error is $1.76 \%$ of the reference with a CPU time of $1.1 \%$ of the reference CPU time. It is exemplified thereby that there is a tremendous gain in CPU time with an acceptable accuracy.

Although, an accuracy estimation of the combined methodology is not performed, due to an unaffordable reference solution for such a case, the separate studies still depicts the robustness of the two algorithms and assumed to transmit to the combined procedure.

\subsection{Combined procedure for CCF}

For the given set of loading parameters the combined homogenisation-cycle jump approach is applied till the damage of any integration point reaches the critical value of $D_{c}$. 60 macroscopic time steps are used for the simulation of a particular macro-cycle along with 30 time steps to describe the micro-cycles. The adaptive cycle jump criterion (eq. (31)) is used to skip several macro-cycles. The number of macro cycles needed to reach a maximum damage of $D_{c}=0.2$ is 18237, with 1000 micro-cycles per macro cycle, i.e a total of $1.8237 \times 10^{7}$ micro-cycles. The same 
problem is repeated, but without any thermal loads and with same temporal discretisation, and the number of macro cycles obtained is 20820 (i.e. a total of $2.082 \times 10^{7}$ micro-cycles) for a maximum damage of $D_{c}=0.2$. Initial 3 macro-cycles are calculated without any cycle jump and thereafter a total of 51 macro-cycles has been effectively computed (using cycle jump) for the whole simulation in both cases. The zero order cumulative plastic strain $p_{0}$ can be calculated on the macro-time scale $t$ as

$$
\dot{p}_{0}=\left(\frac{2}{3} \dot{\varepsilon}_{0}^{p}: \dot{\varepsilon}_{0}^{p}\right)^{1 / 2}=\frac{\dot{r}_{0}}{1-D_{0}} .
$$

The distribution of damage and accumulated plastic strain for both cases (see figs. 5 and 6 ) show that there has been a redistribution due to thermal loads and the region of localisation for both the quantities of interest has changed. Thereby the thermal loads not only influence the number of cycles but also the location of the point susceptible to failure (i.e. the weakest Gauss point).

The evolution of $p_{0}$ and $D_{0}$ at the Gauss points where they are maximum are shown in fig. 7 . Obviously the corresponding Gauss points are different for the purely mechanical and combined thermo-mechanical cases. The evolution of the cumulative plastic strain also shows that for the thermo-mechanical loading, the final value is slightly less than for the purely mechanical case.

The non-linear damage evolutions are more vivid if figs. 7a and $7 \mathrm{~b}$ are zoomed for a particular macro-cycle, as shown in fig. 8 for simulated cycle number 30. The damage evolutions in fig. 8 corresponds to real macro-cycle number 11227 for thermo-mechanical load, and to 13194 for purely mechanical load.

The Gauss points with maximum damage obtained from the results depicted in fig. 5 for both the cases is shown in fig. 9. Stress-strain diagrams for these two points are plotted in figs. 10 and 11. to observe the change behaviour due to fatigue damage. For the relocalised stressstrain diagrams (see figs. 10a and 11a), the residual quantities are added to their homogenised counterparts only at the macro-time points and not interpolated in between. Nevertheless, the relocalised (see figs. 10a and 11a) and homogenised (see figs. 10b and 11b) plots show a significant reduction in the hysteretic area and a significant decrease in the slope (at the elastic region), between the third macro-cycle and the last macro-cycle. Figures 10c and 11c show the residual plot for the micro-cycles corresponding to the first macro-time point of the third and final macro-cycles. These plots are considered to be representative of the other micro-cycles. Although the pure mechanical loading induce a purely elastic stress-strain plot susceptible to damage (see fig. 10c), in the thermo-mechanical case, there exist a hysteretic area along with influence of damage (see fig. 11c). The range of stresses in thermo-mechanical case (see fig. 10) is significantly higher than the purely thermal case (see fig. 11), however the converse is true for the strain range. The final observation that can be made is due to redistribution of the quantities of interest, the dominant stress-strain pair in the purely mechanical case is the $y y$ component which changes to $x x$ component for the thermo-mechanical case.

\subsection{Estimation of numerical expense}

To put into perspective, the reduction of numerical expense, the number of Global Newton iterations and the number of iterations for the non-linear mechanical behaviour can be considered as estimators.

Considering the thermo-mechanical problem, the number of Newton iterations for the simulation of one macro-cycle using the multi-scale method is approximately 196. The total number of cycles, actually simulated is 54 (initial 3 cycles and 51 using the jumping technique), which accounts for a total of approximately 10584 Newton iterations. The total number of iterations (i.e 

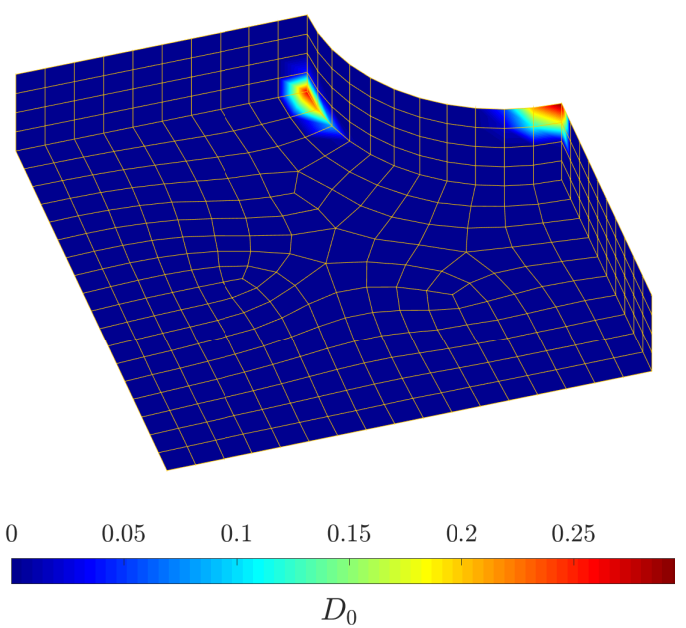

(a) For thermo-mechanical case
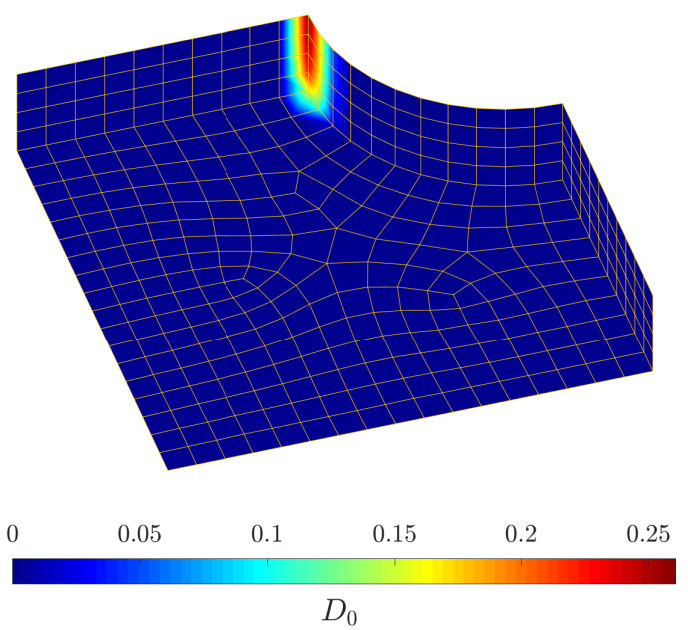

(b) For purely mechanical case

Figure 5: Damage distribution at the end of loading 


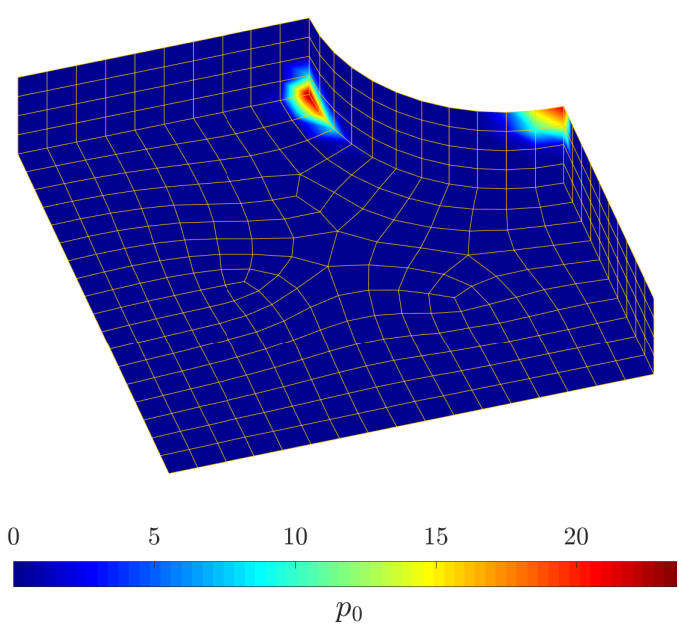

(a) For thermo-mechanical case

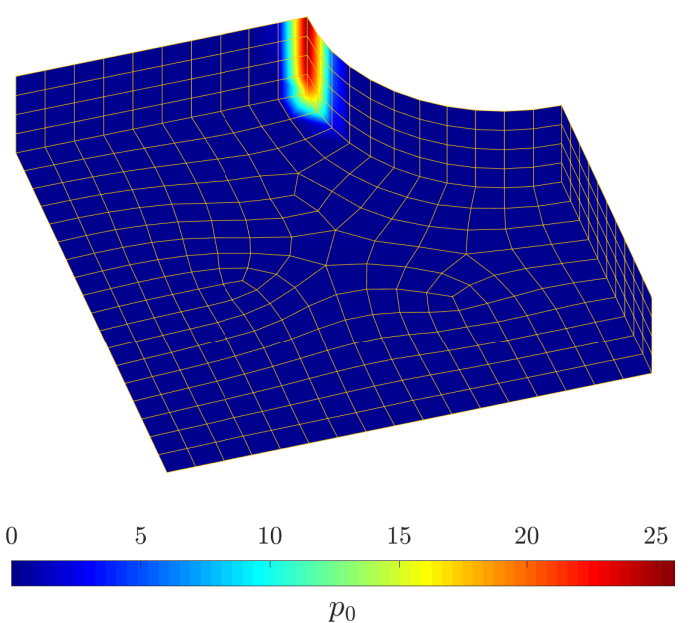

(b) For purely mechanical case

Figure 6: Distribution of cumulative plastic strain at the end of loading 


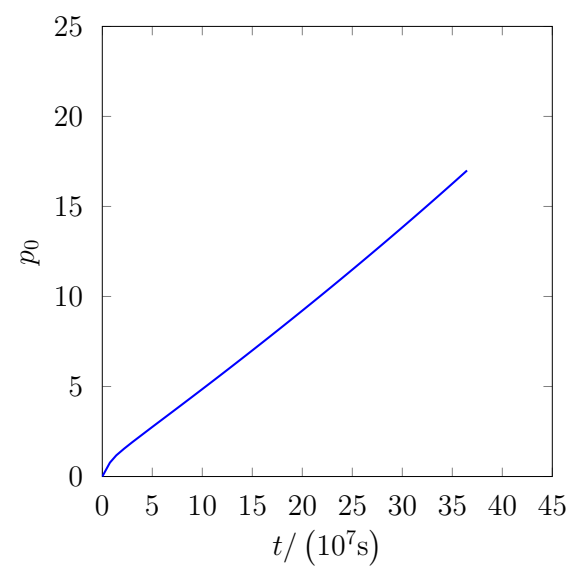

(a) Cumulative plastic strain for thermomechanical loading

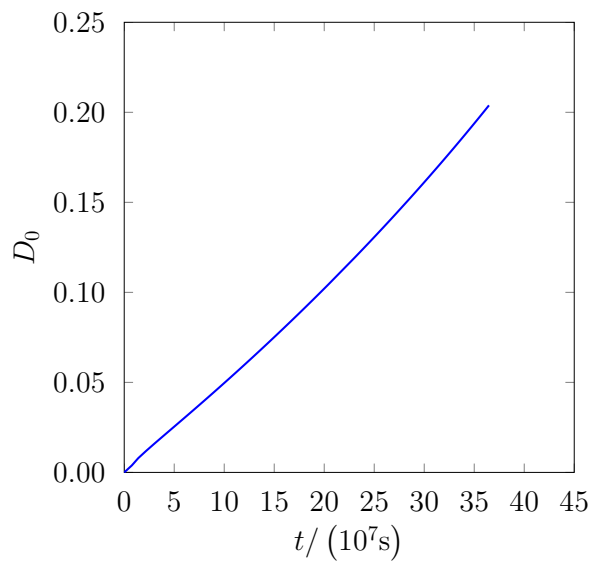

(c) Damage for thermo-mechanical loading

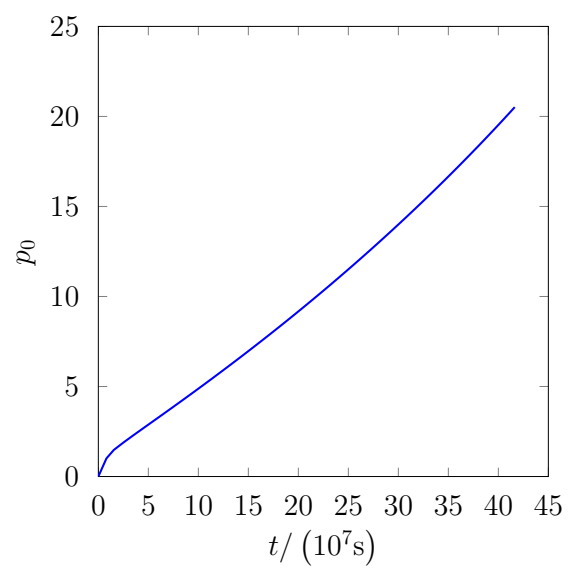

(b) Cumulative plastic strain for purely mechanical loading

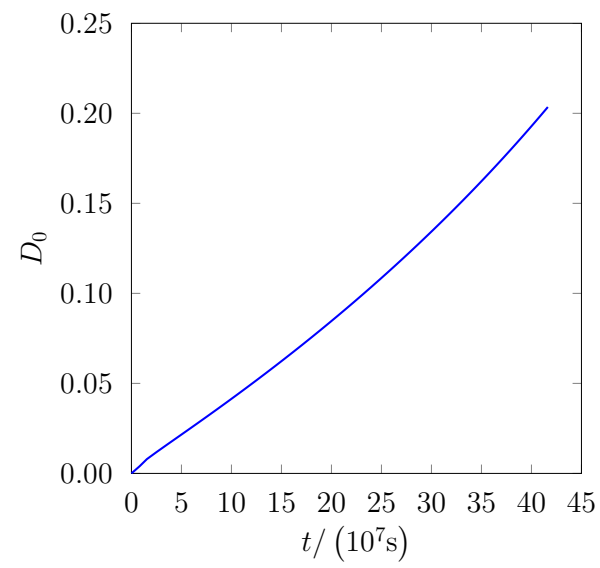

(d) Damage for purely mechanical loading

Figure 7: Evolution of quantities of interest at the respective weakest Gauss points 


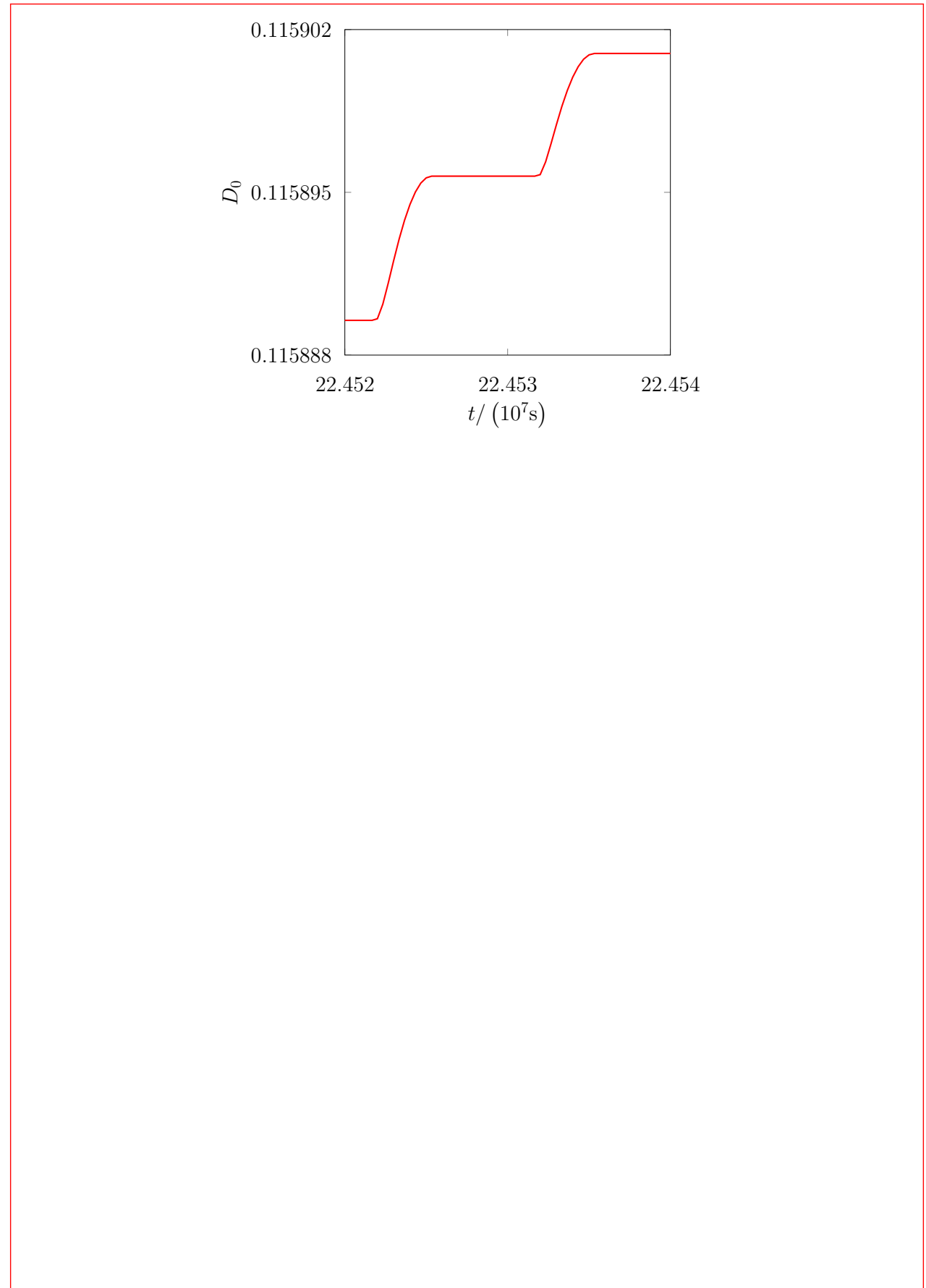

(a) For thermo-mechanical loading (corresponding to actual macro-cycle number 11227)

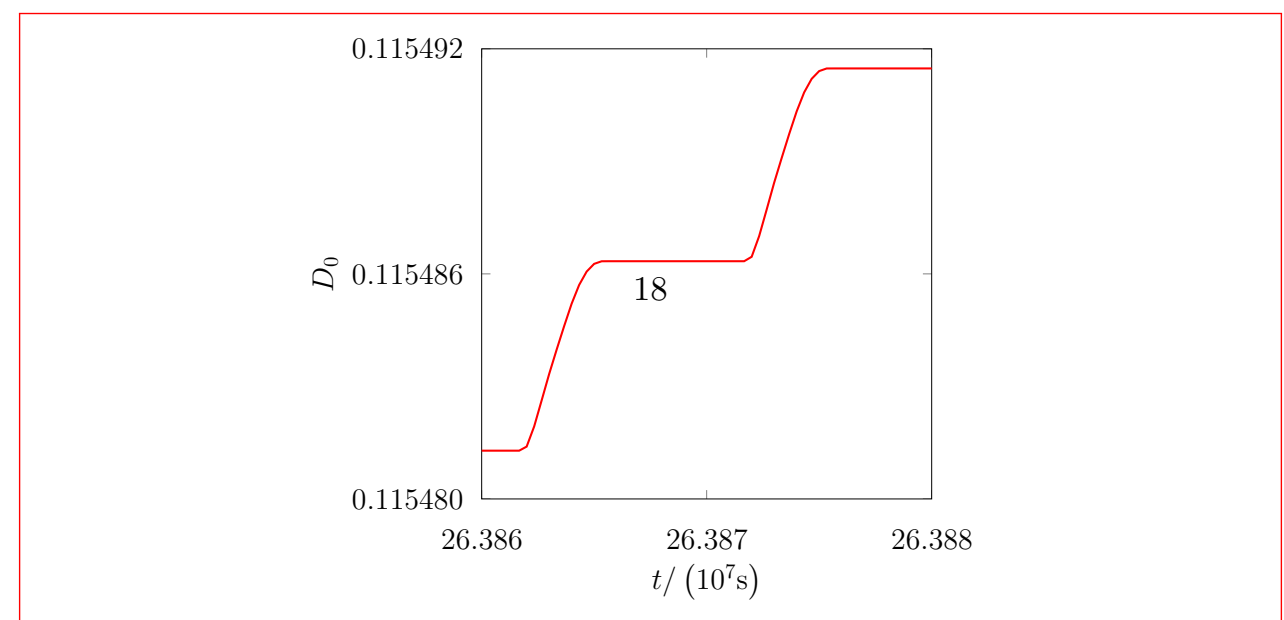




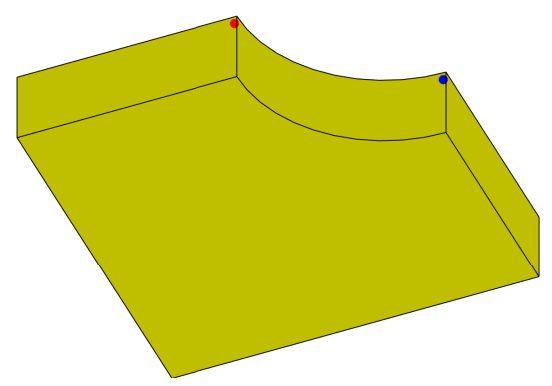

Figure 9: Maximum damaged Gauss points, • for purely mechanical load, • for thermomechanical load

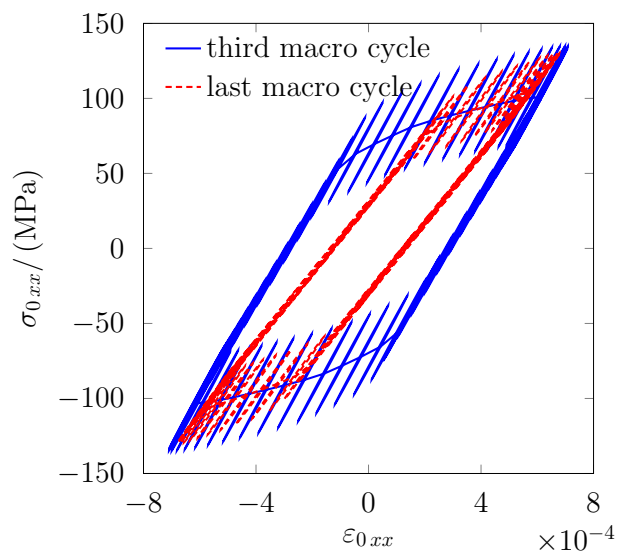

(a) Relocalised behaviour

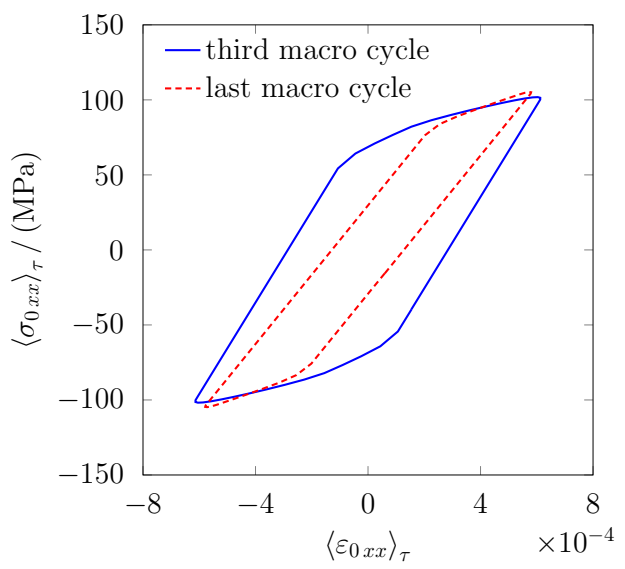

(b) Homogenised behaviour

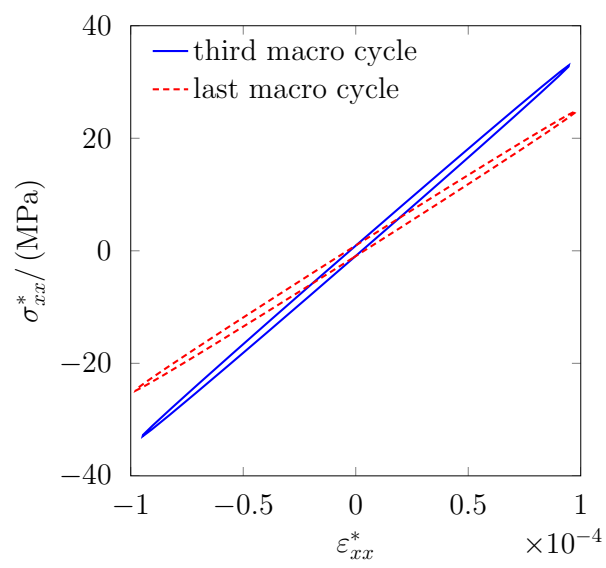

(c) Residual behaviour for a particular microcycle of the corresponding macro-cycle

Figure 10: Stress-strain curves at the most damage Gauss point for the thermo-mechanical case 


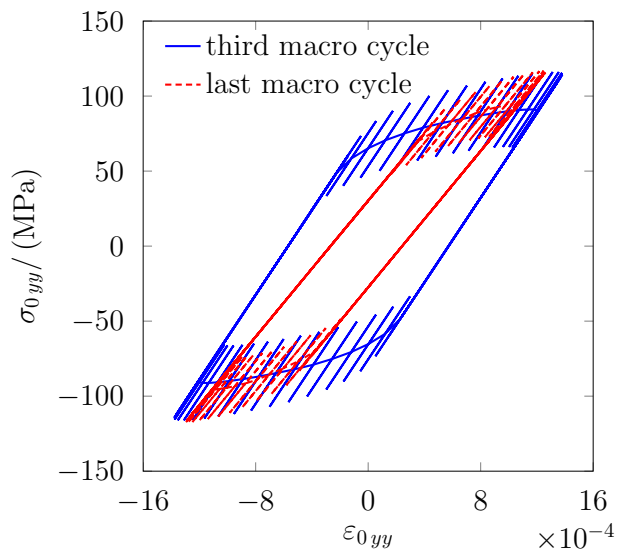

(a) Relocalised behaviour

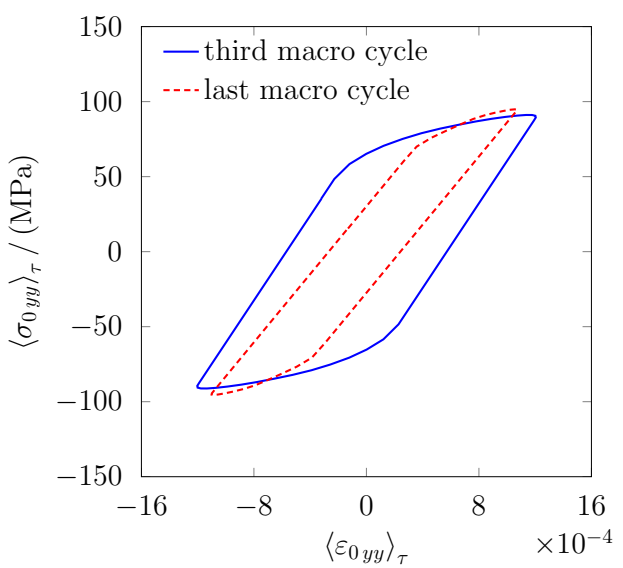

(b) Homogenised behaviour

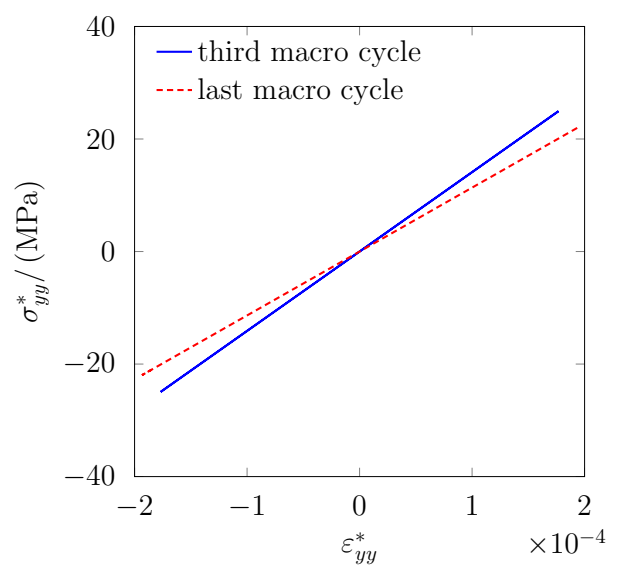

(c) Residual behaviour for a particular microcycle of the corresponding macro-cycle

Figure 11: Stress-strain curves at the most damage Gauss point for the purely mechanical case 


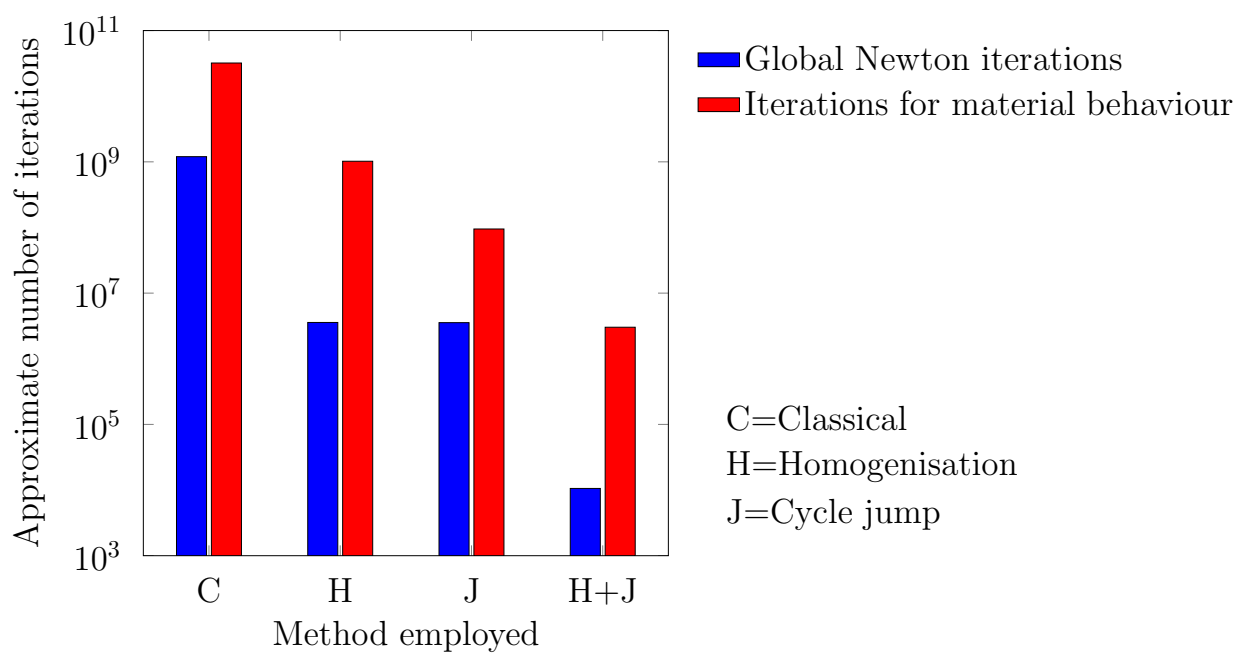

Figure 12: Estimation of approximate iterations for different techniques for the thermomechanical problem

including every Gauss point, every Newton iteration, every time step) required for the solution of the non-linear material law for a macro-cycle is approximately 56000 , i.e. $3 \times 10^{6}$ in total (for 54 cycles). If the complete simulation has to be performed without employing cycle jump method, the number of Newton iterations would be approximately $3.6 \times 10^{6}$ and number of iterations for solving the material behaviour would be approximately $1.0 \times 10^{9}$. If the homogenisation method is also withdrawn, i.e. the complete simulation is performed classically, the number of Newton iteration would approximately be $1.2 \times 10^{9}$ (65753 per macro-cycle) and number of iterations for the non-linear material behaviour would be $3.2 \times 10^{10}\left(1.7 \times 10^{6}\right.$ per macro-cycle). To estimate the cost for the case where only cycle jump is employed for the macro-cycles, each of the computed cycles has to be calculated classically. Assuming the same number of total computed cycles as the unified strategy, a total of $3.5 \times 10^{6}$ Newton iterations would be necessary with a total of $9.5 \times 10^{7}$ iterations for the non-linear material behaviour. These values have been plotted in fig. 12 for a better pictorial understanding of the numerical expenses.

The convergence behaviour of a particular macro-cycle will be different depending on whether it is computed classically or by multi-scale homogenisation. The average Newton iterations per time step for the classical method is estimated to be 2.19 while using the multi-scale it is 3.2. As far as the iterations needed for the non-linear material behaviour is concerned, it is estimated to be at an average of 933 per time step for the multi-scale computation and 58 per time step for the classical computation. The inference herein is that a less number of iterations are needed for convergence at a given time step (both for global equilibrium as well as for local behavioural laws) in case of the classical method than for the multi-scale method.

\section{Conclusion}

A unified framework based on temporal homogenisation and cycle jump technique has been proposed herein to simulate thermo-mechanical combined cycle fatigue. A continuum based damage model has been used to quantify the loss of load carrying capacity. Both the involved frameworks have been verified separately with classical technique and the accuracies were found 
to be appreciable with extreme time savings. The proposed unified framework has been used to simulate till the point of crack initiation for a thermo-mechanical loading and the results have been compared with a purely mechanical loading. The influence of the thermal loading is not only on the number of surviving load cycles but also on the region of damage localisation. Although not addressed in this current work, experimental comparisons indeed remains a perspective of this research work.

\section{Acknowledgment}

The authors wish to thank for grants from Carnot Institute Ingénierie@Lyon (project MURMUR).

\section{References}

[1] E. C. Aifantis. The physics of plastic deformation. Int J Plasticity, 3(3):211-247, 1987.

[2] D. Aubry and G. Puel. CCF modelling with use of a two-timescale homogenization model. Procedia Eng, 2(1):787-796, 2010.

[3] D. Aubry and G. Puel. Two-timescale homogenization method for the modeling of material fatigue. IOP Conference Series: Materials Science and Engineering, 10:012113, June 2010.

[4] Z. P. Bažant and G. Pijaudier-Cabot. Measurement of characteristic length of nonlocal continuum. J Eng Mech, 115(4):755-767, 1989.

[5] S. Bhamare, T. Eason, S. Spottswood, S. R. Mannava, V. K. Vasudevan, and D. Qian. A multi-temporal scale approach to high cycle fatigue simulation. Comput Mech, 53(2):387400, aug 2013.

[6] M. Bhattacharyya, A. Fau, R. Desmorat, S. Alameddin, D. Néron, P. Ladevèze, and U. Nackenhorst. A kinetic two-scale damage model for high-cycle fatigue simulation using multitemporal LATIN framework. Eur J Mech A-Solid, 77:103808, 2019.

[7] M. Bhattacharyya, A. Fau, U. Nackenhorst, D. Néron, and P. Ladevèze. A multi-temporal scale model reduction approach for the computation of fatigue damage. Comput Methods Appl Mech Eng, 340:630 - 656, 2018.

[8] R. Billardon. Etude de la rupture par la mécanique de l'endommagement. PhD thesis, University Paris 6, 1989. in French.

[9] F. Bogard, P. Lestriez, and Y. Q. Guo. Numerical modeling of fatigue damage and fissure propagation under cyclic loadings. Int J Damage Mech, 17(2):173-187, jul 2008.

[10] J. L. Chaboche and P. M. Lesne. A non-linear continuous fatigue damage model. Fatigue Fract Eng M, 11(1):1-17, 1988.

[11] J.-Y. Cognard and P. Ladevèze. A large time increment approach for cyclic viscoplasticity. Int J Plasticity, 9(2):141-157, 1993.

[12] D. Cojocaru and A. M. Karlsson. A simple numerical method of cycle jumps for cyclically loaded structures. Int J Fatigue, 28:1677-1689, 2006.

[13] W. Cui. A state-of-the-art review on fatigue life prediction methods for metal structures. $J$ Mar Sci Tech-Japan, 7(1):43-56, 2002. 
[14] A. Devulder, D. Aubry, and G. Puel. Two-time scale fatigue modelling: application to damage. Comput Mech, 45:637-646, 2010.

[15] A. Fatemi and L. Yang. Cumulative fatigue damage and life prediction theories: a survey of the state of the art for homogeneous materials. Int J Fatigue, 20(1):9-34, 1998.

[16] J. Fish, M. Bailakanavar, L. Powers, and T. Cook. Multiscale fatigue life prediction model for heterogeneous materials. Int J Numer Meth Eng, 91(10):1087-1104, 2012.

[17] J. Fish and C. Oskay. A nonlocal multiscale fatigue model. Mech Adv Mater Struc, 12(6):485-500, nov 2005.

[18] T. Guennouni and D. Aubry. Réponse homogénéisée en temps de structures sous chargements cycliques (in French). Comptes rendus de l'Académie des sciences. Série 2, Mécanique, Physique, Chimie, Sciences de l'univers, Sciences de la Terre, 303(20):1765$1768,1986$.

[19] M. Jirásek. Nonlocal damage mechanics. Rev Eur Génie Civ, 11(7-8):993-1021, 2007.

[20] L. M. Kachanov. In time to rupture in creep conditions. Izviestia Akademii Nauk SSSR, Otdelenie Tekhnicheskikh Nauk, 8:26-31, 1958. in Russian.

[21] J. Lemaitre. Coupled elasto-plasticity and damage constitutive equations. Comput Methods Appl Mech Eng, 51(1):31-49, 1985.

[22] J. Lemaitre. A course on damage mechanics. Springer, Berlin, 1996.

[23] J. Lemaitre and J. L. Chaboche. Mechanics of Solid Materials. Cambridge University Press, 1990.

[24] J. Lemaitre and R. Desmorat. Engineering Damage Mechanics: Ductile, Creep, Fatigue and Brittle Failures. Springer, 2005.

[25] J. Lemaitre and I. Doghri. Damage 90: a post processor for crack initiation. Comput Methods Appl Mech Eng, 115(3):197-232, 1994.

[26] J. Lemaitre and I. Doghri. Damage 90: a post processor for crack initiation. Comput Methods Appl Mech Eng, 115:197-232, 1994.

[27] M. P. Lesne and S. Savalle. An efficient cycles jump technique for viscoplastic structure calculations involving large number of cycles. In D. R. J. Owen, E. Oñate, and E. Hinton, editors, Proceedings of the 2nd International Conference on Computational Plasticity, pages 591-602. Pineridge Press, Ltd., Swansea, 1989.

[28] C. Oskay and J. Fish. Fatigue life prediction using two-scale temporal asymptotic homogenization. Int $J$ Numer Meth Eng, 61:329-359, 2004.

[29] C. Oskay and J. Fish. Multiscale modeling of fatigue for ductile materials. Int J Multiscale Com, 2(3):329-354, 2004.

[30] G. Puel and D. Aubry. Material fatigue simulation using a periodic time-homogenisation method. Eur J Comput Mech, 21(3-6):312-324, 2012.

[31] G. Puel and D. Aubry. Efficient fatigue simulation using periodic homogenization with multiple time scales. Int J Multiscale Com, 12(4):291-318, 2014. 
[32] M. Samal, M. Seidenfuss, and E. Roos. A comparative assessment of local and nonlocal damage models for prediction of fracture behavior during mixed-mode loading. Procedia Eng, 55:493 - 498, 2013. 6th International Conference on Creep, Fatigue and Creep-Fatigue Interaction.

[33] J. Schijve. Fatigue of Structures and Materials. Springer Netherlands, 2008.

[34] B. Sun, Y.-L. Xu, Q. Zhu, and Z. Li. Auto-adaptive multiblock cycle jump algorithm for fatigue damage simulation of long-span steel bridges. Fatigue Fract Eng M, 42(4):919-928, dec 2018.

[35] S. Suresh. Fatigue of materials. Cambridge University Press, Cambridge, 2 edition, 2001.

[36] Q. Yu and J. Fish. Temporal homogenization of viscoelastic and viscoplastic solids subjected to locally periodic loading. Comput Mech, 29(3):199-211, Sep 2002. 